\title{
Shifting attention into and out of objects: Evaluating the processes underlying the object advantage
}

\author{
JAMES M. BRown AND Hope I. DenNeY \\ University of Georgia, Athens, Georgia
}

\begin{abstract}
Visual cuing studies have been widely used to demonstrate and explore contributions from both object- and location-based attention systems. A common finding has been a response advantage for shifts of attention occurring within an object, relative to shifts of an equal distance between objects. The present study examined this advantage for within-object shifts in terms of engage and disengage operations within the object- and locationbased attention systems. The rationale was that shifts of attention between objects require object-based attention to disengage from one object before shifting to another, something that is not required for shifts of attention within an object or away from a location. One- and two-object displays were used to assess object-based contributions related to disengaging and engaging attention within, between, into, and out of objects. The results suggest that the "object advantage" commonly found in visual cuing experiments in which shifts of attention are required is primarily due to disengage operations associated with object-based attention.
\end{abstract}

We constantly shift attention from one place to another, and from one object to another, as we look about and interact with our environment. This seemingly effortless behavior involves complex contributions from and interactions between the perception, attention, and eye movement systems. Of particular interest here are contributions to this behavior from attention systems that are thought to operate independently from location- and object-based frames of reference (see, e.g., Leek, Reppa, \& Tipper, 2003; Reppa \& Leek, 2003; Serences, Liu, \& Yantis, 2005; Tipper, Weaver, Jerreat, \& Burak, 1994). Whether shifts of attention are location based or object based, they could be described as involving three logical steps or processes associated with visual selective attention: engaging, disengaging, and shifting (Posner, 1980). ${ }^{1}$ To shift attention from one location or object to another, attention needs to disengage or reorient from the location or object where it is currently deployed (but see Vecera \& Flevaris, 2005, and our General Discussion). The present study explores engaging, disengaging, and shifting attention within the location- and object-based systems.

A common way to measure contributions from the location- and object-based systems is to use a visual cuing paradigm (e.g., that of Egly, Driver, \& Rafal, 1994), in which attention is first drawn to a location or object by flashing a cue, and the reaction time (RT) is then measured for a shift to a subsequently presented target. RTs are faster when the cue and target appear at the same location (valid cue) than when they appear at different locations (invalid cue). The increase in RT with invalid cues is an indicator of the processing time required to disengage and shift attention from the cue to the target. The location- and objectbased contributions are assessed by comparing invalid cue conditions in which a shift of attention is required either within an object or between objects. A consistent finding in the literature (and in the present study) is that RTs are faster for within-object shifts than for shifts of an equal distance between objects (see Avrahami, 1999; Egly et al., 1994; Egly, Rafal, Driver, \& Starrveveld, 1994; Lavie \& Driver, 1996; Law \& Abrams, 2002). Why and how the advantage for within-object shifts (or the disadvantage for between-object shifts) occurs has been a topic of considerable research and d ebate (Avrahami, 1999; Davis, Driver, Pavani, \& Shepherd, 2000; Goldsmith \& Yeari, 2003; Lamy \& Egeth, 2002; Shomstein \& Yantis, 2002, 2004; see the General Discussion below for a detailed discussion of alternative theoretical conceptualizations in the context of the present study). Lamy and Egeth's (2002) study using detection, discrimination, and flanker interference tasks illustrated that shifting attention may be a boundary condition for producing object-based effects. We explore this boundary condition further by asking what it is about shifting attention that produces object-based effects.

From a location-based perspective, within-object and between-object shifts involve disengaging from one location, shifting, and engaging on a new location. The only difference is that in some cases these actions are executed within an object and in others between objects. From an object-based perspective, within-object shifts involve disengaging attention from one place on an object, shifting,

J. M. Brown, jmbrown@uga.edu 
and then engaging it on another place within the same object. A topic of critical importance to the present study, however, is what occurs during between-object shifts. If engaging and disengaging attention from a location and from an object involve different or separate processes, then engaging and disengaging attention from an object is more than simply engaging and disengaging attention from a location. From this perspective, between-object shifts would involve separate engage, disengage, and shift operations for both the location-based and object-based systems. Thus, the object advantage found in cuing studies may result from additional processes of the object-based system that are involved in disengaging from the invalidly cued object, engaging on the object the target appears in, or both. In addition to Lamy and Egeth's (2002) findings, two other studies have specifically suggested this idea. Vecera (1994) held the within-object distance constant while testing a between-object distance that was shorter than the within-object distance. RTs for between-object shifts were shorter when the between-object distance was less than the within-object distance, but they were still not as fast as RTs for within-object shifts. Conversely, Brown, Breitmeyer, Leighty, and Denney (2006) held the between-object distance constant while testing a withinobject distance that was greater than the between-object distance. They found that RTs for within-object shifts increased when the within-object distance was more than the between-object distance, but were still shorter than RTs for between-object shifts. In both studies, the object advantage persisted, even though it was reduced in magnitude relative to control conditions in which the withinand between-object distances were equal. Together, these results suggested to us that something specific to shifts of attention between objects makes them slower than shifts within objects. A common thread linking these studies is that responses were slower when attention had to disengage from an object before shifting than when it did not, irrespective of distance.

Visual cuing studies often compare the costs for invalid cues that require shifts of attention within an object with the costs for shifts between objects. We are unaware of any cuing studies directly comparing shifts from an object to a location, from a location to an object, and from a location to another location (but see Leek, Reppa, \& Tipper's [2003] study on inhibition of return). Our goal was to determine whether the object advantage found in cuing studies is driven by attention's having to disengage from an object and/or having to engage on a new object during between-object shifts by comparing conditions in which attention shifts within objects, between objects, "out of" objects (object-to-location), "into" objects (location-toobject), and between two locations (location-to-location).

\section{EXPERIMENT 1}

In order to test for separate processes of engaging and disengaging attention from a location and from an object, we used one- and two-object stimuli (Figures 1A-1H). The two-object condition was important because we needed to establish that a typical object advantage could be found under the present experimental conditions, and also because it provided a performance baseline against which specific one-object conditions (described in detail below) could be compared, as a means of separating out the contributions of the engage and disengage operations of the location- and object-based systems. The two-object stimuli were used to show a basic attention-cuing effect, in which valid RTs are expected to be faster than invalid RTs, and to establish an object advantage, in which invalid within-object RTs (for an example, see Figure 1A) are expected to be faster than invalid between-object RTs (Figure 1B). To help distinguish between the one-object and two-object conditions, we will refer to the two-object valid condition as two-valid and the two-object invalid conditions as two-within and between-object. The specific one-object conditions, with their purposes and predicted outcomes, are listed next.

\section{One-Object Valid Conditions}

Valid-in. When the cue and target appeared in the same place at one end of the single object presented in a trial, it was called a valid-in trial because both cue and target appeared in an object (Figure 1C). The purpose of this condition was twofold. First, this condition was necessary in order to show a basic attention-cuing effect by comparing RTs in this condition with those in one-object invalid conditions. Second, it was important to show that RTs on one-object valid trials were no different from those on two-object valid trials, so that we could make direct comparisons of the one- and two-object conditions. Since the sequences of events were identical in all valid trials, if one-object valid-in RTs were slower or faster than twoobject valid RTs, it would suggest that performance was influenced by the number of objects in the display.

Valid-out. When the cue and target appeared in the same place on the opposite side of the screen from the single object presented in a trial, it was called a validout trial because both cue and target appeared outside of any object. The cue and target positions were the same as when they appeared in an object, just on the side of the screen where no object was present (Figure 1F). The main purpose of this condition was to ensure that any increases in RTs found for one-object invalid conditions in which targets appeared outside of an object could not simply be explained by that fact. RTs in this condition were expected to be the same as, or possibly faster than, valid-in and two-valid RTs. The rationale for why they might be faster was that, from a signal-to-noise perspective, the cue and target might be somewhat easier to detect without object contours nearby.

\section{One-Object Invalid Conditions}

Within-object. When the cue and target appeared at opposite ends of the single object, it was a one-object within-object trial (Figure 1D; this condition will hereafter be referred to as the one-within condition). The sequence of events would be identical to the two-within condition, but with only one object present. RTs were expected to be similar to two-within RTs because the engage and disengage operations would be identical. 


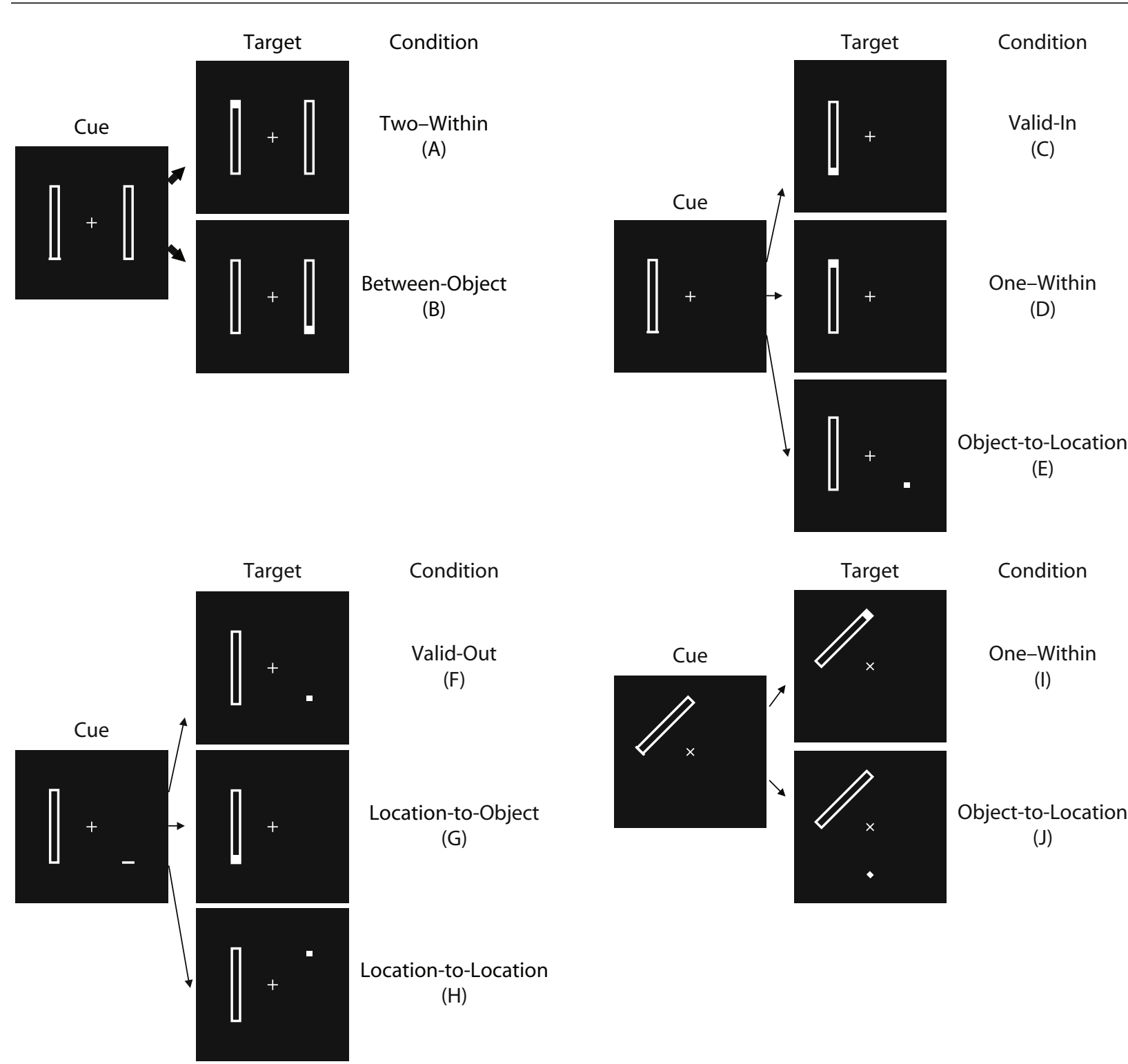

Figure 1. An illustration of the different conditions of our experiments. (A and B) Two-object invalid conditions. (C-H) The various one-object cue-target conditions with objects presented in the vertical orientation. (I and J) Two one-object invalid conditions with the object tilted to the right.

Location-to-location. When the cue and target appeared on the side opposite the single object presented in a trial, this was a location-to-location trial (Figure $1 \mathrm{H}$; hereafter, $l o c \rightarrow l o c)$. The cue and target positions were the same as in one-within trials, except they appeared on the side of the screen opposite where the object appeared. This condition provided a situation in which object-based engage and disengage operations would not be involved, because the cue and target appeared outside of any objects. RTs were expected to be similar to one-within and two-within RTs because, as in those conditions, object-based attention would not have to disengage from an object in order to shift, nor would it engage on another object after shifting.

Location-to-object. When the cue appeared on the side opposite the single object presented in a trial and the target appeared in the end of the object closest to the cue, this was a location-to-object trial (Figure 1G; hereafter, $l o c \rightarrow o b j)$. This condition would not require attention to disengage from an object before shifting, just as in both within-object conditions and the loc $\rightarrow$ loc condition, but unlike those conditions, it would entail engaging on an object after shifting. Thus, if RTs in this condition were greater than those in both of the within-object conditions and the loc $\rightarrow$ loc condition, this could be taken as evidence that an object-based engage operation occurred after shifting.

Object-to-location. When the cue appeared in one end of the single object presented in a trial and the target appeared in the closest comparable position on the empty side of the display, this was an object-to-location trial 
(Figure 1E; hereafter, $o b j \rightarrow l o c$ ). This condition would require attention to disengage from an object before shifting, making it most similar to the between-object condition. It is different from that condition, though, in not requiring that attention engage on another object after shifting. Thus, if RTs were greater in this condition than in both within-object conditions and the loc $\rightarrow$ loc condition, it would be evidence of an object-based disengage operation. In addition, if RTs were similar to those in the between-object condition, it would provide evidence that the typical object advantage is driven by the necessity of disengaging object-based attention from the cued object before shifting.

Comparisons of RTs in this condition and in the loc $\rightarrow$ obj condition would also provide information on the relative influences of object-based engage and disengage operations. If RTs were greater for obj $\rightarrow$ loc than for loc $\rightarrow$ obj shifts, this would indicate that disengaging has a greater influence than engaging object-based attention. Likewise, RTs in the opposite direction would indicate greater influence for engaging than for disengaging object-based attention. No difference in RTs would indicate that both operations have similar influence.

\section{Method}

\section{Participants}

A total of 30 University of Georgia undergraduates (15 male, 15 female) participated for introductory psychology course credit. All participants had normal or corrected-to-normal vision, were classified as right-handed according to the Annett Handedness Scale, and reported no history of attention deficit disorder.

\section{Stimuli and Apparatus}

Stimulus presentation and data collection were carried out using commercially available software (SuperLab Pro) running on a PC with a VGA monitor. Responses were collected from a response box that interfaced with the computer. The participants sat in a darkened room $70 \mathrm{~cm}$ from the monitor and used a chinrest.

All stimuli were white on a black background. The fixation cross was $0.76^{\circ} \times 0.76^{\circ}$ in size. Objects (whether one or two) were centered $2.39^{\circ}$ from fixation and subtended $5.33^{\circ} \times 0.57^{\circ}$. The lines constructing the objects subtended $0.19^{\circ}$. Targets consisted of a solid square $\left(0.57^{\circ}\right.$ on each side) that either filled one end of an object or, when it appeared outside the object in one-object conditions, occupied the same location on the opposite side of the screen. It was the same shade of white as the lines making up the objects. Cues consisted of a slightly whiter line, $0.57^{\circ}$ long and $0.19^{\circ}$ thick, which again appeared either at the end of an object or in the same location on the side opposite a single object.

\section{Procedure}

All one- and two-object conditions were randomly intermixed within each block during the experiment. Following a brief introduction in which the various trial conditions were shown once, participants completed 9 practice trials before starting the experiment. Trials were presented in six blocks of 120 trials with a short break between blocks, for a total of 720 trials.

Each block of 120 trials consisted of $72(60 \%)$ valid trials, in which the cue and target appeared in the same location; 24 (20\%) invalid trials, in which the cue and target did not appear in the same location; and $24(20 \%)$ catch trials, in which no target appeared. The 72 valid trials consisted of 24 two-object trials and 24 trials each in which a single object appeared on the left or on the right of the screen. The 24 invalid trials consisted of 4 trials from each of the six invalid conditions: two-within, between-object, one-within, $\mathrm{loc} \rightarrow \mathrm{loc}, \mathrm{loc} \rightarrow \mathrm{obj}$, and obj $\rightarrow \mathrm{loc}$.

An example of the sequence of events is depicted in Figure 2 for a two-object valid trial. Each trial began with a white fixation cross at the center of the screen. After a participant initiated a trial with a keypress, either one or two objects appeared with the fixation cross for $1,000 \mathrm{msec}$, followed by a cue that appeared for $50 \mathrm{msec}$. After $150 \mathrm{msec}$, the target appeared until the participant responded or until $1,500 \mathrm{msec}$ had elapsed. On catch trials, no target appeared. After either a response or $1,500 \mathrm{msec}$, there was a 500 -msec blank interval before the fixation stimulus returned, signaling the next trial. RTs were measured from the onset of the target until a response was made. Participants received a warning screen for RTs less than $150 \mathrm{msec}$ or false alarms on catch trials.

\section{Results and Discussion}

RTs less than $150 \mathrm{msec}$ and greater than $1,000 \mathrm{msec}$ $(2 \%)$ were trimmed from all analyses. The mean false alarm rate on catch trials was $4 \%$. Mean RTs were calculated for each participant and each condition for the subsequent analyses (see Figure 3). In the discussion below, RTs in parentheses indicate mean RT $\pm S E$.

We first ran tests for the basic cuing and object advantage effects, separately for the two- and one-object conditions. A one-way ANOVA on two-object valid and invalid RTs indicated a significant 29 -msec cuing effect $[F(1,29)=20.06, p<.001]$, with valid RTs $(326 \pm$ $10 \mathrm{msec}$ ) faster than invalid RTs ( $355 \pm 13 \mathrm{msec})$. A oneway ANOVA on two-within and between-object RTs indicated a significant 13 -msec object advantage $[F(1,29)=$ $8.47, p<.007]$ due to two-within RTs $(348 \pm 12 \mathrm{msec})$

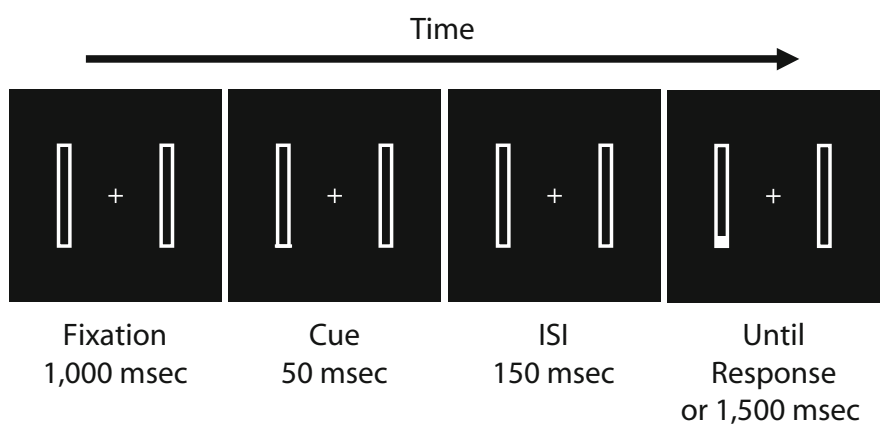

Figure 2. An example of a vertical two-object valid trial, illustrating the sequence of events. 


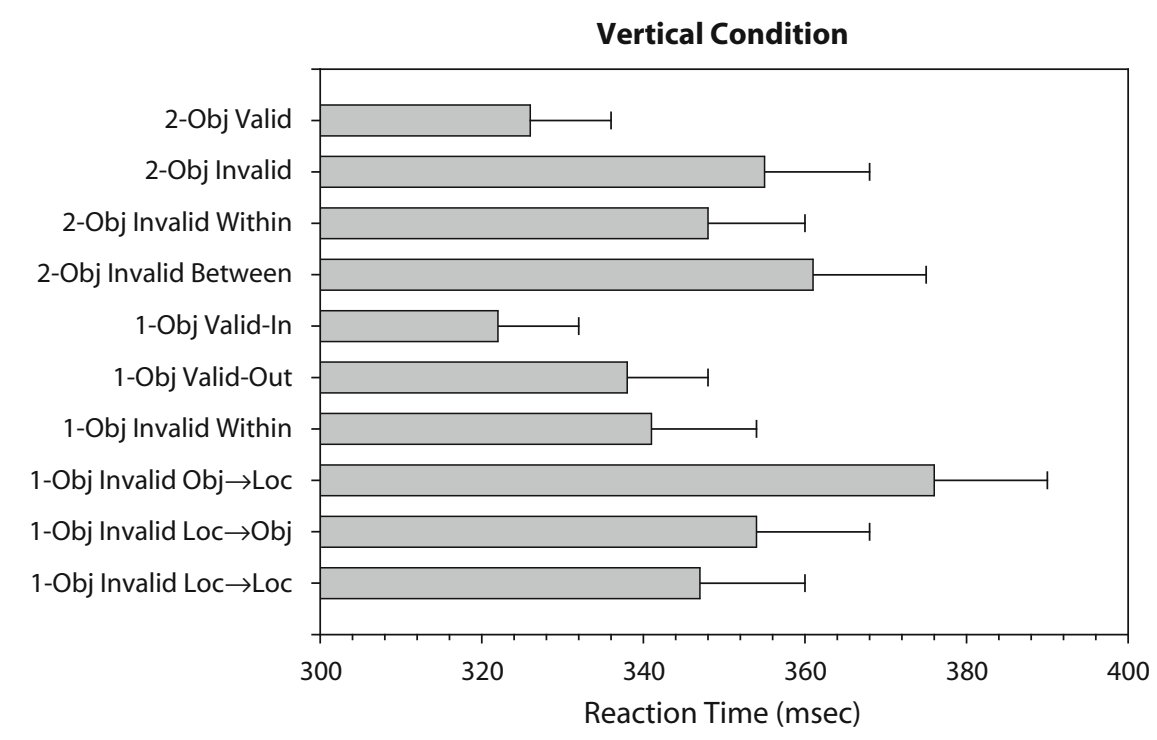

Figure 3. Reaction times for all vertically oriented two- and one-object valid and invalid conditions (Experiment 1).

being faster than between-object RTs (361 $\pm 14 \mathrm{msec})$. To test for a cuing effect with the one-object stimuli, we ran a one-way ANOVA on one-object valid versus invalid RTs. To do this, we collapsed across both one-object valid conditions (-in and -out) and the four one-object invalid conditions (one-within, loc $\rightarrow$ loc, loc $\rightarrow$ obj, and obj $\rightarrow l o c$ ). An overall 25-msec cuing effect was evident in which the one-object valid RTs (330 $\pm 10 \mathrm{msec})$ were significantly faster than the invalid RTs $(355 \pm 13 \mathrm{msec})[F(1,29)=$ $21.51, p<.001]$.

A one-way ANOVA on RTs for the three valid conditions allowed us to test whether valid RTs were influenced by the presence of an object (e.g., valid-in vs. valid-out) or the presence of a second object (e.g., valid-in vs. twovalid). Post hoc comparisons indicated that the significant effect of condition $[F(2,58)=15.42, p<.001]$ was due to the two-valid $(326 \pm 10 \mathrm{msec})$ and valid-in $(322 \pm$ $10 \mathrm{msec}$ ) RTs being shorter than the valid-out RTs (338 \pm $10 \mathrm{msec})$. All post hoc tests throughout the study used Newman-Keuls tests and a significance level of $p<.05$. The valid-in and two-valid RT means were equivalent, indicating that the presence or absence of a second object did not influence valid responses to targets appearing in an object. Finding longer valid-out RTs was unexpected. We had predicted similar (or possibly faster) RTs for this condition. We will discuss the valid-in and valid-out conditions further following Experiment 2 and in the General Discussion.

We had predicted that the three invalid conditions in which object-based attention neither had to disengage from an object to shift nor engage on another object after shifting would be similar, and this prediction was confirmed through a one-way ANOVA: The one-within $(341 \pm 12 \mathrm{msec})$, two-within $(349 \pm 12 \mathrm{msec})$, and loc $\rightarrow$ loc $(347 \pm 13 \mathrm{msec})$ RTs were not different from each other $[F(2,58)=0.99, p>.38]$.
A one-way ANOVA comparing RTs in all one-object invalid conditions (one-within, loc $\rightarrow \mathrm{obj}, \mathrm{obj} \rightarrow \mathrm{loc}$, and loc $\rightarrow$ loc) was significant $[F(3,87)=15.05, p<.001]$. The condition of primary interest was the obj $\rightarrow$ loc condition, because only in this condition would attention have to disengage from an object before shifting. As predicted, obj $\rightarrow$ loc RTs (376 $\pm 14 \mathrm{msec}$ ) were significantly longer than those in the one-within $(341 \pm 12 \mathrm{msec})$, loc $\rightarrow$ loc $(347 \pm 13 \mathrm{msec})$, and loc $\rightarrow$ obj $(354 \pm 14 \mathrm{msec})$ conditions, with no differences between the latter three. These results suggest that disengaging object-based attention from an object before shifting plays a primary role in the object advantage and that engaging on an object after a shift of attention does not. If engaging on an object after a shift did play an important role, loc $\rightarrow$ obj RTs should have been significantly longer than loc $\rightarrow$ loc RTs.

Our finding that valid-out RTs were longer than validin RTs might suggest that we take caution in interpreting the results of the obj $\rightarrow$ loc condition as support for the importance of disengaging object-based attention from an object before a shift, because the target appeared outside of an object in the valid-out condition, too. However, if the longer valid-out RTs indicated generally slowed responses to targets that appear by themselves rather than in an object, this slowing influence should have been evident for all invalid conditions in which the target appeared by itself. For example, in the loc $\rightarrow$ loc condition, in which the target appeared by itself, RTs should have been longer than those in one-within trials, in which the target appeared within an object; this was not the case, however. These comparisons suggest that, whatever the reason that valid-out RTs were longer than valid-in RTs, that factor likely did not contribute to the differences in RTs between the various one-object invalid conditions (see the Results below for Experiments 2 and 3). 
Although these results were encouraging, we should make one observation about our stimuli and procedure. We chose to use only vertically oriented stimuli for our test because of the number of one- and two-object conditions we wanted to test in a within-subjects design. Using such stimuli allowed us to keep the experiment at a reasonable length for participants to finish in a single visit. Another fact that contributed to this decision was that, in previous two-object experiments in which we had used both vertical and horizontal stimuli (Brown et al., 2006), we had never found any effect of object orientation on the object advantage. Although these were legitimate reasons for using only vertical stimuli, the between-object and obj $\rightarrow$ loc conditions that produced the longest RTs were also two of the three conditions in which attention had to shift from one visual field/cerebral hemisphere to another (e.g., from the right visual field/left hemisphere to the left visual field/right hemisphere, or vice versa). All within-object shifts (with one object or with two) and loc $\rightarrow$ loc shifts took place within a visual field. It was possible, then, that this factor could have had some influence on the pattern of results. Our second experiment was designed to rule out this possibility.

\section{EXPERIMENT 2}

To test whether the pattern of one- and two-object results in Experiment 1 was in some way influenced by attention having to shift from one visual field to another, the stimuli we used in Experiment 2 were rotated $45^{\circ}$ either clockwise (for examples, see Figures 1I and 1J) or counterclockwise, with orientation a between-subjects factor. In this experiment, shifts for all one- and two-object valid and invalid conditions always occurred within a visual field and never involved crossing the vertical meridian. If the trends of results changed remarkably in these condi- tions, that finding would support the visual field hypothesis and go against our hypotheses concerning engaging and disengaging attention. If the trends of results remained the same, this would replicate Experiment 1 and confirm the involvement we hypothesized of object-based engage and disengage operations.

\section{Method}

\section{Participants}

A total of 60 University of Georgia undergraduates (30 male, 30 female) participated for introductory psychology course credit. All participants had normal or corrected-to-normal vision, were classified as right-handed according to the Annett Handedness Scale, and reported no history of attention deficit disorder.

\section{Stimuli and Apparatus}

The details of the stimuli and apparatus remained the same as in Experiment 1, with the exception that all stimuli were presented tilted $45^{\circ}$ either clockwise or counterclockwise from vertical.

\section{Procedure}

All procedural details were identical to those in Experiment 1, except that one group of 30 participants ( 15 male, 15 female) were presented stimuli tilted to the right, and another group of 30 (also 15 male, 15 female) were presented stimuli tilted to the left.

\section{Results and Discussion}

RTs less than $150 \mathrm{msec}$ and greater than $1,000 \mathrm{msec}$ $(2 \%)$ were trimmed from all analyses. The mean false alarm rate on catch trials was once again 4\%. Mean RTs were again calculated for each condition for the subsequent analyses (see Figure 4).

Initial ANOVAs on the various comparisons made in Experiment 1, using bar orientation as a between-subjects factor, revealed no main effects of orientation or interactions of orientation with other factors, so the results of the following analyses are collapsed across orientations. A one-way ANOVA on two-object valid and invalid RTs

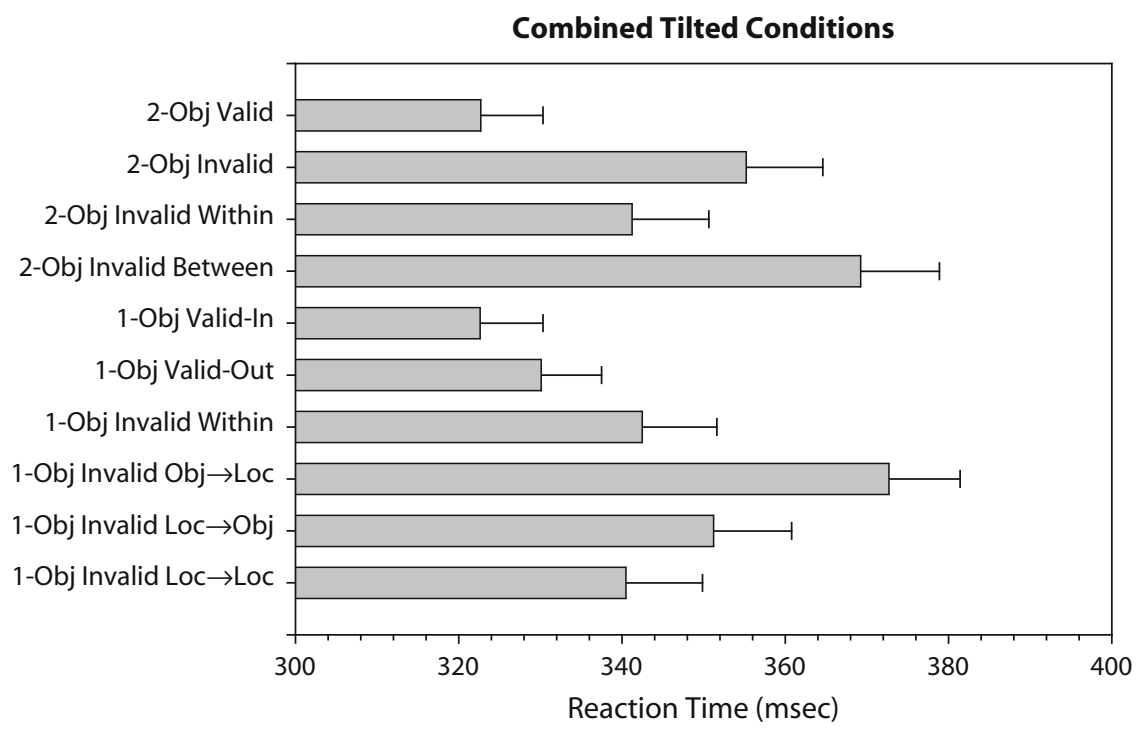

Figure 4. Reaction times, collapsed across left- and right-tilted orientations, for all two- and one-object valid and invalid conditions (Experiment 2). 
indicated a significant $22-\mathrm{msec}$ cuing effect $[F(1,59)=$ $72.45, p<.001]$, with valid RTs $(323 \pm 8 \mathrm{msec})$ faster than invalid RTs $(355 \pm 9 \mathrm{msec})$. A one-way ANOVA on two-within and between-object RTs indicated a significant 28 -msec object advantage $[F(1,59)=75.52, p<$ $.001]$ due to within-object RTs $(341 \pm 9 \mathrm{msec})$ being faster than between-object RTs $(369 \pm 10 \mathrm{msec})$. To test for a cuing effect with the one-object stimuli, we ran a one-way ANOVA on one-object valid versus invalid RTs, as in Experiment 1, and found overall a significant 26msec cuing effect, with the one-object valid RTs (326 \pm $7 \mathrm{msec})$ faster than the invalid RTs $(352 \pm 9 \mathrm{msec})$ $[F(1,59)=93.81, p<.001]$. A comparison of the results from Experiment 1, in which half of the invalid conditions involved crossing the vertical meridian, with those here indicates that crossing the vertical meridian was not a significant factor influencing the earlier results.

A one-way ANOVA comparing RTs for the valid-in, validout, and two-valid conditions was significant $[F(2,118)=$ $15.87, p<.001]$. Post hoc comparisons showed no difference in RTs between the valid-in $(323 \pm 8 \mathrm{msec})$ and two-valid $(323 \pm 8 \mathrm{msec})$ conditions, as predicted. This is important, because both experiments show that valid responses to targets appearing within an object are not influenced by the number of objects in the display. However, valid-out RTs were again slightly, but significantly, longer $(330 \pm 7 \mathrm{msec})$ than those in the other two valid conditions. Why valid-out RTs were significantly longer in both experiments, despite the smaller difference $(7 \mathrm{msec})$ in this experiment, is not clear. One speculation is that bottom-up, stimulus-driven information may automatically draw attention to the single object in one-object displays during the second that the object is visible before the cue-target sequence begins. This attention to the single object might contribute to the slight processing advantage of a cued object over a cued empty location, resulting in slightly shorter valid-in RTs (but see the Overall Analyses section below and Experiment 3).

Replicating the results of Experiment 1, one-within (342 $\pm 9 \mathrm{msec}$ ), two-within ( $341 \pm 9 \mathrm{msec}$ ), and loc $\rightarrow$ loc $(340 \pm 9 \mathrm{msec})$ RTs were no different from each other $[F(2,118)=0.14, p>.87]$. Again, this result supported our prediction that RTs in these three invalid conditions should be similar because object-based attention would neither have to disengage from an object to shift nor engage on another object after shifting.

A one-way ANOVA comparing RTs in all one-object invalid conditions (one-within, loc $\rightarrow \mathrm{obj}, \mathrm{obj} \rightarrow \mathrm{loc}$, and loc $\rightarrow$ loc) was significant $[F(3,177)=30.54, p<.001]$. Again, the obj $\rightarrow$ loc condition was of primary interest because only in this condition would object-based attention have to disengage from an object before shifting. As in Experiment 1, the obj $\rightarrow$ loc condition had the longest RTs of any condition. $\mathrm{Obj} \rightarrow \mathrm{loc}$ RTs $(373 \pm 9 \mathrm{msec})$ were significantly longer than one-within (342 $\pm 9 \mathrm{msec})$, loc $\rightarrow$ loc $(340 \pm 9 \mathrm{msec})$, and loc $\rightarrow$ obj $(351 \pm 10 \mathrm{msec})$ RTs. Note particularly that RTs in the loc $\rightarrow$ obj condition were significantly longer than loc $\rightarrow$ loc RTs. This finding is discussed further in the next section. As predicted, the one-object invalid condition that produced the lon- gest RTs in both experiments was the obj $\rightarrow$ loc condition, which required object-based attention to disengage from an object before shifting.

\section{Overall Analyses}

Three additional analyses were conducted, incorporating the data from both experiments and using orientation as a between-subjects factor with three levels, vertical (Experiment 1) and tilted left and tilted right (Experiment 2). First, a 3 (orientation) $\times 6$ (invalid conditions) ANOVA on RTs from all invalid conditions showed no main effect of orientation $[F(2,87)=0.77, p>.55]$ or interaction with invalid condition $[F(6,261)=0.56$, $p>.75]$, but there was a significant main effect of invalid condition $[F(3,261)=44.37, p<.001]$. RTs for the between-object (368 $\pm 8 \mathrm{msec})$ and obj $\rightarrow \operatorname{loc}(375 \pm$ $7 \mathrm{msec}$ ) conditions were significantly longer than those in all other conditions, but not significantly different from each other. The two-within $(345 \pm 7 \mathrm{msec})$, one-within $(343 \pm 7 \mathrm{msec})$, and loc $\rightarrow$ loc $(344 \pm 8 \mathrm{msec})$ conditions produced the shortest RTs and were not different from each other. RTs in the loc $\rightarrow$ obj $(353 \pm 8 \mathrm{msec})$ condition were between these extremes and different from those in all other conditions.

A 3 (orientation) $\times 3$ (valid conditions) ANOVA on RTs from the two-valid, valid-in, and valid-out conditions showed no main effect of orientation $[F(3,177)=30.54$, $p<.001]$, but there was a significant main effect of valid condition $[F(2,174)=30.87, p<.001]$ and a significant interaction $[F(4,174)=2.40, p<.052]$. Two-valid $(324 \pm 6 \mathrm{msec})$ and valid-in $(323 \pm 6 \mathrm{msec})$ RTs were not different from each other and were shorter than validout ( $333 \pm 6 \mathrm{msec})$ RTs. As is evident in Table 1, the primary reasons for the orientation interaction are the overall shorter RTs and smaller RT differences across valid conditions for the tilted-right orientation. Although valid-out RTs were significantly longer than valid-in RTs for this orientation, this difference was less than in the other two orientations, and the valid-out and two-valid RTs were not different from each other. For the other two orientations, valid-out RTs were significantly longer than both valid-in and two-valid RTs. We should emphasize the tenuous nature of both the speculations earlier and those below about the overall slower valid-out RTs, since valid-out RTs were not different from two-valid RTs (in which the target appears in an object) for the tilted-right orientation.

The final analysis was a cost analysis of cue conditions across orientations. A cost analysis normalizes responses to targets in the one-object invalid conditions, relative

Table 1

Mean Reaction Times (in Milliseconds) for the Valid Conditions of Each Orientation in Experiments 1 (Vertical) and 2 (Tilted Left or Right)

\begin{tabular}{lccc}
\hline & Vertical & Left & Right \\
\hline Valid & 326 & 333 & 315 \\
Valid-in & 322 & 333 & 313 \\
Valid-out & 338 & 343 & 319 \\
\hline
\end{tabular}

Note-The standard error for all conditions is $\pm 10 \mathrm{msec}$. 
both to each other and to the two-object conditions, by subtracting the appropriate valid condition as a baseline. Thus, the cost indicates how much additional time was necessary to make a response to a target appearing at a particular position when a shift of attention to that position was necessary versus when it was not. As such, validin RTs were subtracted from RTs in the two conditions in which the target appeared in the object (one-within and loc $\rightarrow$ obj). Valid-out RTs, on the other hand, were subtracted from those in the two conditions in which the target appeared outside the object (obj $\rightarrow$ loc and loc $\rightarrow$ loc). Two-object costs were calculated by subtracting two-valid RTs from the two-within and between-object RTs. A 3 (orientation) $\times 6$ (cue condition) mixed-design ANOVA conducted on the costs, with orientation the betweensubjects factor, showed a significant main effect of cue condition $[F(5,435)=36.9, p<.001]$ but no main effect of orientation $[F(2,87)=0.31, p>.74]$ or interaction $[F(10,435)=1.12, p>.33]$. Table 2 summarizes the costs $( \pm S E)$ over the different invalid conditions.

Cost comparisons across cue conditions were consistent with the RT analyses, but one result raised a concern relating back to the valid-in/valid-out RT difference. Consistent with the RT analyses, shifts out of an object (i.e., between-object and obj $\rightarrow$ loc shifts) produced the greatest costs of any of the conditions, and the presence of a second object did not influence the time to shift either within (i.e., two-within vs. one-within) or out of (i.e., betweenobject vs. obj $\rightarrow$ loc) an object. In addition, loc $\rightarrow$ obj shift costs were different from those in all other conditions, suggesting a possible influence of object-based attention engaging on an object after a shift.

One result, however - the finding that costs for loc $\rightarrow$ loc shifts were different from those in all other conditionsdid raise a concern that related back to the valid-in/validout RT difference. A comparison of the loc $\rightarrow$ loc condition with both within-object conditions suggested that shifts within an object were somehow slower than those between two locations. The reason for our concern was that, whatever advantage an object may have afforded responses to validly cued targets within it, once a cue had drawn attention to the object and a shift of attention was required within it, the shift seemed to take more time than did shifts of the same distance between two positions outside an object. This potentially theoretically provocative finding suggested the need for a closer examination of the sequence of events in each trial from the perspectives of engage, disengage, and shift operations. In particular, because loc $\rightarrow$ loc and obj $\rightarrow$ loc costs are calculated using valid-out RTs as the baseline, what should be made of the slightly but significantly $(7 \mathrm{msec})$ shorter RTs for validly cued targets that appeared inside rather than outside an object? Although we will describe two possible explanations, it should be noted that we believe that neither (or both) of the processes we propose may have contributed to the results (see, e.g., Experiment 3). First, the effect might be akin to an object superiority effect, in which the presence of an object facilitates perceptual processing of a target appearing within it (Weisstein \& Harris, 1974), even in comparison with a target appearing alone (Williams \& Weisstein, 1978). This could explain why valid-in and two-valid RTs could be faster relative to the valid-out condition. However, as noted above, valid-out and two-valid RTs were not different with the tilted-right orientation, which raises doubts about any speculations concerning the valid-in/valid-out differences.

Another possibility comes from Vecera and Behrmann's (2001) biased competition perspective on object segregation and attention. According to their account, object segregation and selection for processing involve a competition between bottom-up information from the physical stimulus and top-down information related to an observer's goals. It seems less likely that top-down influences could have determined the results here, because both the instructions and the cue-target probability always indicated that targets were most likely to appear at cued locations, and this probability was the same whether the location was in or out of an object. From this perspective, the facilitation of responses for the valid-in relative to the valid-out condition may have been due to bottom-up information, consistent with an object superiority account. During the preview period of each trial, bottom-up cues (i.e., the presence of the single object) may have automatically drawn attention to the object (and to both objects when there were two). When a cue appeared in an object, the process of engaging on the position of the cue was facilitated because attention had already been drawn to the object. When the cue appeared outside of an object, the location-based engage operation associated with the cue would have had to, in a sense, overcome attention already engaged at the location of the object during the preview period. Thus, responses were facilitated when valid cue-target sequences appeared within rather than outside an object because attention had been drawn beforehand to the single object. As one reviewer noted, the valid-in/valid-out difference might be considered a bias cost associated with the cue when it appears outside a single object. To address this issue, a final, control experiment was run with no objects in the display.

\section{EXPERIMENT 3}

The main purpose of this experiment was to measure RTs to validly and invalidly cued targets appearing in

Table 2

Average Costs for All Invalid Conditions, Collapsed Across Orientations

\begin{tabular}{|c|c|c|c|c|c|}
\hline \multicolumn{2}{|c|}{ Two Objects } & \multicolumn{4}{|c|}{ One Object } \\
\hline Within & Between & One-Within & $\mathrm{Obj} \rightarrow \mathrm{Loc}$ & $\mathrm{Loc} \rightarrow \mathrm{Obj}$ & $\mathrm{Loc} \rightarrow \mathrm{Loc}$ \\
\hline $20 \pm 3$ & $43 \pm 4$ & $20 \pm 3$ & $41 \pm 3$ & $30 \pm 3$ & $10 \pm 3$ \\
\hline
\end{tabular}


open space, devoid of any potentially biasing influence of a single nearby object. This measurement would allow for comparisons of (1) no-object valid RTs with the valid-in and valid-out RTs from Experiment 2, and (2) the costs for shifts in open space, with no objects present, with those in the various one- and two-object invalid conditions from Experiment 2.

\section{Method}

\section{Participants}

A total of 20 University of Georgia undergraduates (12 male, 8 female) participated for introductory psychology course credit. All participants had normal or corrected-to-normal vision, were classified as right-handed according to the Annett Handedness Scale, and reported no history of attention deficit disorder.

\section{Stimuli and Apparatus}

The no-object condition was run under the same viewing conditions as in Experiment 2 with cues and targets appearing at the same four positions relative to each other and to the fixation cross.

\section{Procedure}

The total of 240 trials consisting of $144(60 \%)$ validly cued, 48 $(20 \%)$ invalidly cued, and $48(20 \%)$ catch trials. As before, invalid targets only appeared at the two positions closest to the cues. All other procedural details were identical to those in Experiment 2, except for the absence of objects in the display.

\section{Results and Discussion}

RTs less than $150 \mathrm{msec}$ and greater than $1,000 \mathrm{msec}$ $(2 \%)$ were trimmed from all analyses. The mean false alarm rate on catch trials was $5 \%$. Valid RTs were averaged over position, and invalid RTs were averaged over all shift directions between cues and targets. A one-way ANOVA for the no-object valid and invalid RTs indicated a significant 15 -msec cuing effect $[F(1,19)=10.41, p<$ $.004]$, with valid RTs $(320 \pm 10 \mathrm{msec})$ faster than invalid RTs $(335 \pm 10 \mathrm{msec})$. Costs were calculated as before, with RTs to validly cued targets at a position subtracted from RTs to invalidly cued targets at that same position. No-object RTs and costs were then compared with those from Experiment 2 (collapsed across left/right orientation) using independent $t$ tests (two-tailed). Only the Experiment 2 data were used for comparison because the positions of cues and targets relative to fixation and to each other were identical in both experiments. No-object valid RTs $(320 \pm 10 \mathrm{msec})$ were not different from either the valid-out RTs [331 $\pm 7 \mathrm{msec} ; t(78)=0.80, p=.43]$ or the valid-in RTs [323 $\pm 8 \mathrm{msec} ; t(78)=0.25, p=.81]$. This lack of a difference between the no-object valid RTs and both the valid-in and valid-out RTs suggests that, although the result was statistically significant, the slightly longer (7 msec) valid-out RTs in Experiment 2 likely did not reflect the presence of the single object, because there was no object present in this control experiment to influence RTs. The present RT results and a comparison of them with the RTs in Experiment 2 suggest that our concern and speculations about the differences between validin and valid-out RTs were unwarranted. If valid-out RTs had been influenced by the presence of the single object, removing that object should have removed the influence, and no-object RTs should then have been different from valid-out RTs; this was not found. Similarly, if the single object had facilitated valid responses within it, a difference should be found between valid-in and no-object valid RTs, and this difference was not found, either.

Cost comparisons (see Table 3) indicated that shift times for no-object invalid trials $(15 \pm 5 \mathrm{msec})$ were not different from those in the loc $\rightarrow$ loc $[10 \pm 4 \mathrm{msec} ; t(78)=$ $-0.69, p=.49]$, one-within [20 $\pm 4 \mathrm{msec} ; t(78)=0.67$, $p=.51]$, and two-within $[18 \pm 3 \mathrm{msec} ; t(78)=0.53, p=$ $.60]$ conditions. These results suggest that caution should be used when interpreting, and speculating about, the seemingly faster shift times in the overall analyses above for the loc $\rightarrow$ loc than for the within-object condition (see Table 2). If, as we speculated earlier, shifts in open space were somehow faster than those within an object, costs should then have been less in the no-object than in the within-object condition, but this was not the case. Relative to the no-object condition, shift times were actually longer for the loc $\rightarrow$ obj $[29 \pm 4 \mathrm{msec} ; t(78)=1.99, p=$ $.05], \mathrm{obj} \rightarrow \operatorname{loc}[43 \pm 3 \mathrm{msec} ; t(78)=4.47, p=.001]$, and between-object [47 $\pm 5 \mathrm{msec} ; t(78)=3.60, p=.001]$ conditions. Finding costs in those conditions that were greater than in the no-object condition is also consistent with the overall analyses in Experiment 2, in which those costs were also greater than those in the loc $\rightarrow$ loc and within-object conditions.

In light of the results of the overall analyses above and of the comparisons with the no-object condition, the comparisons of the results in the loc $\rightarrow$ obj condition with those in the loc $\rightarrow$ loc and both within-object conditions suggest possible influences from the engage operation in objectbased attention. Although the operations associated with engaging on and disengaging from the cue and shifting would all be the same for the loc $\rightarrow$ obj and loc $\rightarrow$ loc conditions, those associated with engaging on the target after a shift would be different, since an object-based engage operation would only become involved when the target appeared in the object. Thus, engaging on a position after a shift would be less costly than engaging on an object, because only location-based attention would be involved

Table 3

Average Costs for the No-Object Condition (Experiment 3) and the Invalid Conditions of Experiment 2, Collapsed Across Left and Right Orientations

\begin{tabular}{|c|c|c|c|c|c|c|}
\hline \multicolumn{2}{|c|}{ Two Objects } & \multicolumn{4}{|c|}{ One Object } & \multirow{2}{*}{$\begin{array}{c}\text { No Object } \\
(\text { Loc } \rightarrow \text { Loc) }\end{array}$} \\
\hline Within & Between & One-Within & $\mathrm{Obj} \rightarrow \mathrm{Loc}$ & $\mathrm{Loc} \rightarrow \mathrm{Obj}$ & $\mathrm{Loc} \rightarrow \mathrm{Loc}$ & \\
\hline $18 \pm 4$ & $47 \pm 5$ & $20 \pm 4$ & $43 \pm 3$ & $29 \pm 4$ & $10 \pm 4$ & $15 \pm 5$ \\
\hline
\end{tabular}


for the position, but both location- and object-based attention would be involved for the object. Although future psychophysical tests of this account are needed in order to test it directly, the account does seem reasonable, and it receives support from neuroimaging. Using an event-related fMRI paradigm, Arrington, Carr, Mayer, and Rao (2000) found greater activity across a number of brain areas during the deployment of object-based rather than locationbased attention, and never vice versa. They interpreted these findings as indicating that object-based deployment "is gained by adding to or supplementing the brain activity needed to allocate attention to a similarly situated but fuzzily bounded spatial region defined by environmental coordinates rather than object shape" (p. 111). A similar argument could be made for why the costs associated with the loc $\rightarrow$ obj condition could be greater than those for the within-object conditions. Again, only in the loc $\rightarrow$ obj condition would object-based attention have to engage on an object after a shift. In the within-object conditions, object-based attention would already be engaged on the object because of the cue, but in the loc $\rightarrow$ obj condition the object-based engage operation would not be activated until the target appeared in the object. Because the distance across which shifts occurred was held constant, the greater costs for the loc $\rightarrow$ obj condition could be related to the need for the object-based engage operation after a shift. From Arrington et al.'s perspective, this might be attributed to the additional brain activation associated with object-based attention becoming involved in the loc $\rightarrow$ obj condition once the target appears in the object, in comparison with the within-object conditions, in which similar brain activation would already have been involved from the time the cue appeared in the object.

Finally, costs were less for loc $\rightarrow$ obj shifts than for either obj $\rightarrow$ loc or between-object shifts, but the costs in the latter two conditions were similar. This particular finding might be considered evidence against the involvement of object-based engage operations, because they would have been involved in the loc $\rightarrow$ obj and between-object conditions, but not in the obj $\rightarrow$ loc condition. There are a few explanations of why this might occur. First, the method used here to measure these influences (RTs) may not have been sufficiently sensitive to separate out the influences of disengage and engage operations when both were involved. This could certainly have been the case if objectbased disengage operations have a greater influence on performance than do engage operations. Thus, the costs of engage operations are evident in the absence of disengage operations in the loc $\rightarrow$ obj condition, and the costs of disengage operations are evident in the absence of engage operations in the obj $\rightarrow$ loc condition, but the involvement of disengage operations in the between-object condition may be such that they obscure evidence of the engage operations. Finally, it is also possible that these speculations about engage operations may be incorrect.

To summarize, the between-object and obj $\rightarrow$ loc conditions consistently produced the longest RTs and greatest costs of all of the invalid conditions, and the former conditions were the only ones in which object-based atten- tion should have had to disengage from an object before shifting. The results of Experiment 2 ruled out shifting attention from one visual field to another as an account of the results of Experiment 1, showed that the basic findings of Experiment 1 are replicable, and strongly implicated object-based disengage operations as an important contributor to the disadvantage found for shifts of attention between rather than within objects in cuing studies. Finally, the greater costs for the loc $\rightarrow$ obj condition in comparison with the loc $\rightarrow$ loc and any of the within conditions may reflect the involvement of engage operations in object-based attention.

\section{GENERAL DISCUSSION}

The present study explored the engage and disengage operations of both object- and location-based forms of attention and the role those operations play in the object advantage commonly found in attention-cuing studies. Previous research showed that shifting attention might be critical for producing object effects (Lamy \& Egeth, 2002) and that the object advantage in cuing studies is robust when between-object distance (Vecera, 1994) and withinobject distance (Brown et al., 2006) are manipulated. A common thread linking these studies is that responses are slower when attention must disengage from an object before shifting than when it does not have to. This result led to our hypothesis that disengage operations associated with object-based attention play a primary role in producing the object advantage in cuing studies. Our results support this hypothesis and also indicate that the "object advantage" in cuing studies may more accurately be described as a disadvantage associated with attention shifting out of, or away from, an object (see Lamy \& Egeth, 2002, for a similar disadvantage argument).

We interpret our results as building on and expanding Lamy and Egeth's (2002) finding that shifting attention is an essential task requirement for producing object-based effects. A shift of attention was required for all invalid trials in our experiments. By varying where attention shifted from and where it shifted to, we could directly compare RTs and costs in one- and two-object invalid conditions. As noted in the introduction, from the perspective of locationbased attention, all of the invalid conditions tested should have produced the same results, because attention always had to disengage from the cue location before shifting to the target location, and the cue-to-target distance was the same in all invalid conditions. Any differences found between the various one- and two-object invalid conditions must, therefore, be attributed to influences (or lack of influences) of object-based attention. Following an evaluation of these influences in terms of the engage and disengage operations of the object- and location-based attention systems, we will consider spreading-attention (Abrams \& Law, 2000; Avrahami, 1999; Brown et al., 2006), biased-competition (Vecera, 1994, 2000; Vecera \& Behrmann, 2001; Vecera \& Flevaris, 2005), and prioritization (Shomstein \& Yantis, 2002, 2004) perspectives on the object advantage and on our results. 


\section{Shifting Between Two Objects Versus Shifting From an Object to a Location}

Object-based attention would have to disengage from an object before shifting in both the between-object and obj $\rightarrow$ loc conditions. In the between-object condition, object-based attention would also have to engage on a new object after shifting away from the original object. The fact that we found no differences in RTs or costs between these conditions is one indicator suggesting that engaging object-based attention on an object after a shift may not be a major contributor to the typical object disadvantage, but that disengaging attention from an object before shifting is.

\section{Shifting From an Object to a Location Versus From a Location to an Object}

A comparison of these one-object conditions allowed us to directly assess the difference between disengaging from an object and disengaging from a location. If objectand location-based attention operate independently (see, e.g., Leek et al., 2003; Reppa \& Leek, 2003; Tipper et al., 1994), the processes of engaging and disengaging attention within these systems would most likely operate independently as well. If disengaging from a cued object involves both disengaging location-based attention from the cued object's location and disengaging object-based attention from the cued object, an additional disengage operation would be associated with object-based attention for obj $\rightarrow$ loc shifts relative to loc $\rightarrow$ obj shifts. The longer RTs and greater costs for the obj $\rightarrow$ loc and between-object conditions relative to the loc $\rightarrow$ obj condition are another indicator that disengaging object-based attention is a primary factor.

\section{Shifting From One Location to Another Versus From an Object to a Location}

A comparison of the loc $\rightarrow$ loc and obj $\rightarrow$ loc conditions provided an opportunity to examine the influence of object-based disengage operations without any potential influences of object-based engage operations occurring after a shift, because in both conditions attention shifted to a location, and only in the obj $\rightarrow$ loc condition would disengaging object-based attention before shifting have been necessary. The consistently longer RTs and greater costs for the obj $\rightarrow$ loc than for the loc $\rightarrow$ loc condition also support the idea that disengaging object-based attention is the primary contributor to the object disadvantage.

\section{Shifting From One Location to Another Versus From a Location to an Object}

Comparing the loc $\rightarrow$ loc with the loc $\rightarrow$ obj condition provided another way to assess the contribution from object-based attention engaging on an object after a shift. This comparison could be made without influences from object-based disengage operations, because attention always shifted from a location outside an object. The greater costs for the loc $\rightarrow$ obj condition suggest an influence of object-based engage operations, although this influence was not noticeable when object-based disengage opera- tions were also involved (e.g., in the between-object and obj $\rightarrow$ loc conditions).

\section{Shifting From One Location to Another Versus Within an Object}

Although location-based attention would be involved in both loc $\rightarrow$ loc and within-object shifts, object-based attention would only be involved in the within-object shift. However, once the cue arrives, there is basically no difference between these conditions in terms of disengage, shift, and engage operations. For the within-object condition, once the cue draws attention to a location in the object, the shift from cue to target occurs within the object (i.e., disengaging object-based attention is not involved), and thus only location-based attention must disengage in order to shift from cue to target location. Likewise, for the loc $\rightarrow$ loc condition, once attention is drawn to the cue location outside the single object, only disengaging location-based attention is required in order to shift to the target location. Thus, no difference was found between the loc $\rightarrow$ loc and within-object RTs, as well as no real difference in shift times between these two conditions, as evidenced by comparisons with the no-object condition of Experiment 3.

\section{Spreading-Attention, Biased-Competition, and Prioritization Perspectives on the Object Advantage}

Spreading-attention, biased-competition, and prioritization perspectives each offer an account of the object advantage, and therefore an account of the faster within-object shifts found here in comparison with the between-object and obj $\rightarrow$ loc conditions. In its own way, each account emphasizes the facilitative influence of attention on processing within an object, a characteristic that distinguishes these perspectives from the disengage perspective.

The spreading-attention perspective attributes the facilitation of within-object shifts to a process of attention moving, radiating, or spreading through an object, guided by that object's contours (Abrams \& Law, 2000; Avrahami, 1999; Brown et al., 2006).

The biased-competition perspective (Vecera, 1994, 2000; Vecera \& Behrmann, 2001; Vecera \& Flevaris, 2005) indicates that bottom-up and top-down biases contribute to the object advantage. In general, these biases compete in the perceptual segregation and organization of the visual field, as well as in the allocation of attention within it. In our one- and two-object displays, bottom-up information would segregate the object(s) from the background, biasing attentional selection toward them and, therefore, constraining the focus of attention relative to empty space (Vecera \& Behrmann, 2001). Although this theory could account for attention being drawn to the object(s) in our experiments, it could not account for the object advantage, because our objects were identical, and bottom-up information would not have biased selection of one object over the other (Vecera, 2000). In our task, the cue was the main attention-biasing factor. According to Vecera (2000), 
a "spatial precue may allow spatial attention to bias attention in a top-down manner" (p. 372); when "spatial attention is summoned to a cued location, attention can spread or move within a closed region more easily than between closed regions" (p. 364; i.e., cuing an object leads to spreading attention within it). "The spatial precue acts to bias object attention toward the cued object, allowing observers to respond faster to targets appearing in the cued object than in the uncued object" (Vecera, 2000, p. 372). In light of our stimuli and task, this perspective seems to incorporate both spreading and prioritization views: The enclosed contours of the cued object facilitate the spread of attention within it in a bottom-up manner, and the cue also biases object-based attention toward the cued object in a top-down manner that could be interpreted as a strategic influence on the allocation of attention to that object over others.

The prioritization perspective also emphasizes a processing advantage within objects (Shomstein \& Yantis, 2004). Object-based selection reflects "an implicit objectspecific attentional prioritization strategy that arises only when multiple locations in the scene must be attended" (Shomstein \& Yantis, 2004, p. 248); "whenever there is more than one object in the scene, regions within an attended object will, by default, be assigned higher priority for visual exploration than other objects" (p. 253). Thus, when attention is not narrowly focused, within-object positions are accorded priority in processing, leading to an object advantage (Shomstein \& Yantis, 2002). As Lamy and Egeth (2002) have noted, however, when Shomstein and Yantis (2002) did not find an object effect with their interference paradigm, the target always appeared at fixation, and thus attention never had to shift in order to accomplish the task. In the one experiment in which they did find an object effect, they modified their paradigm so that shifts of attention were involved in performing the task. Thus, the extent to which the focus of attention and/or shifts in attention contributed to the presence or absence of an object effect is not certain.

A closer comparison of the spreading, biasedcompetition, and prioritization views in terms of the allocation of attention following a cue suggests that little may distinguish them, except perhaps the allocation of attention within an object. The prioritization view defines the allocation of attention in terms of an implicit scanning strategy, whereas both the spreading-attention and biasedcompetition perspectives describe it as a spreading of attention. However, by definition, people would no more be aware of an implicit within-object scanning strategy than of attention spreading within an object. Similarly, whereas spreading attention is thought to occur from the cue to other locations in the cued object, implicit priority given to visual exploration of within-object positions would also be assumed to occur from the cue to other locations in the cued object. Thus, from all of these perspectives, attention facilitates processing within objects in a manner that proceeds from cued to uncued positions.

The different focus of our disengage perspective, on a disadvantage for shifts away from objects rather than an advantage for shifts within them, highlights the difference between our view and the spreading, biased-competition, and prioritization perspectives. Our perspective also expands on Lamy and Egeth's (2002) emphasis on shifting attention by considering the disengagement of attention before and the engagement of attention after shifting. This theory is clearly different from the spreading-attention and biased-competition perspectives, both of which incorporate spreading attention.

It should be acknowledged that the present study was not specifically designed to distinguish between the disengage and prioritization perspectives. However, because the disengage account was the basis for the present experiments, it does have the advantage of incorporating all the findings under one conceptual framework. As noted above, however, the prioritization perspective does provide a reasonable account for the object disadvantages found in our one- and two-object conditions. One anonymous reviewer noted that if within-object locations are always given a higher priority when the cue is partially valid, shifting from an object to a location should be slower than shifting from a location to an object, because in the former case there is a tendency to search the noncued within-object location first. The reviewer also noted that shifting from an object to a location would be slower than shifting from one location to another for the same reason. However, because the cues appeared in locations not occupied by an object in the loc $\rightarrow$ obj and loc $\rightarrow$ loc conditions, the prioritization perspective would seem to have nothing to say about the slower loc $\rightarrow$ obj responses. In order to maintain a prioritization account for the present study, a modification of its defining characteristics would seem to be necessary: When there is only one object in the scene, yet multiple locations must be attended, if attention is drawn away from the object, locations outside the object may receive higher prioritization than locations associated with the object. Such an addition to the prioritization perspective seems necessary in order to account for the longer RTs in the loc $\rightarrow$ obj than in the loc $\rightarrow$ loc condition. However, this addition would also seem to take the prioritization account beyond the realm of object-specific attentional selection, making it a blend of object- and location-based prioritization. This change may or may not fit with the author's intentions.

Finally, it should also be noted that our psychophysical findings are consistent with recent event-related fMRI studies of location- and object-based attention. There is growing evidence that transient activity in slightly different regions of the superior parietal lobule, intraparietal sulcus, and precuneus coincide with shifts of visual attention between (respectively) spatial locations, stimulus features, and objects (Corbetta, Kincade, Ollinger, McAvoy, \& Shulman, 2000; Corbetta \& Shulman, 2002; Serences et al., 2005; Yantis et al., 2002; Yantis \& Serences, 2003). Transient activity associated with shifts of attention across these perceptual dimensions has been interpreted as reorienting signals in order to disengage and shift attention (Serences et al., 2005; Yantis et al., 2002; Yantis \& Serences, 2003) and cause a change in the state of biased competition in other brain regions associated with stimulus and task demands (Serences et al., 2005). Contributions from, 
and interactions between, the transient cortical activity in the regions related to location- and object-based attention shifts may underlie the psychophysically measured differences found across the conditions of the present study.

\section{AUTHOR NOTE}

Correspondence relating to this article may be sent to J. M. Brown, Department of Psychology, University of Georgia, Athens, GA 306023013 (e-mail: jmbrown@uga.edu).

\section{REFERENCES}

Abrams, R. A., \& LAW, M. B. (2000). Object-based visual attention with endogenous orienting. Perception \& Psychophysics, 62, 818-833.

Arrington, C. M., Carr, T. H., Mayer, A. R., \& Rao, S. M. (2000). Neural mechanisms of visual attention: Object-based selection of a region in space. Journal of Cognitive Neuroscience, 12(Suppl. 2), 106-117.

Avrahami, J. (1999). Objects of attention, objects of perception. Perception \& Psychophysics, 61, 1604-1612.

Brown, J. M., Breitmeyer, B. G., Leighty, K. A., \& Denney, H. I. (2006). The path of visual attention. Acta Psychologica, 121, 199-209.

Corbetta, M., Kincade, J. M., Ollinger, J. M., McAvoy, M. P., \& Shulman, G. L. (2000). Voluntary orienting is dissociated from target detection in human parietal cortex. Nature Neuroscience, $\mathbf{3}$, 292-297.

Corbetta, M., \& Shulman, G. L. (2002). Control of goal-directed and stimulus-driven attention in the brain. Nature Reviews Neuroscience, 3, 201-215.

Davis, G., Driver, J., Pavani, F., \& Shepherd, A. (2000). Reappraising the apparent costs of attending to two separate visual objects. Vision Research, 40, 1323-1332.

Egly, R., Driver, J., \& RAfal, R. D. (1994). Shifting visual attention between objects and locations: Evidence from normal and parietal lesion subjects. Journal of Experimental Psychology: General, 123, 161-177.

Egly, R., Rafal, R., Driver, J., \& Starrveveld, Y. (1994). Covert orienting in the split brain reveals hemispheric specialization for objectbased attention. Psychological Science, 5, 380-383.

Goldsmith, M., \& Yeari, M. (2003). Modulation of object-based attention by spatial focus under endogenous and exogenous orienting. Journal of Experimental Psychology: Human Perception \& Performance, 29, 897-918.

LAmy, D., \& Egeth, H. (2002). Object-based selection: The role of attentional shifts. Perception \& Psychophysics, 64, 52-66.

Lavie, N., \& Driver, J. (1996). On the spatial extent of attention in object-based visual selection. Perception \& Psychophysics, 58, 1238-1251.

Law, M. B., \& Abrams, R. A. (2002). Object-based selection within and beyond the focus of spatial attention. Perception \& Psychophysics, 64, 1017-1027.

LeEK, E. C., RepPA, I., \& TipPer, S. P. (2003). Inhibition of return for objects and locations in static displays. Perception \& Psychophysics, 65, 388-395.

Posner, M. I. (1980). Orienting of attention. Quarterly Journal of Experimental Psychology, 32, 3-25.

REPPA, I., \& LEEK, E. C. (2003). The modulation of inhibition of return by object-internal structure: Implications for theories of object-based attentional selection. Psychonomic Bulletin \& Review, 10, 493-502.

Serences, J. T., LiU, T., \& Yantis, S. (2005). Parietal mechanisms of attentional control: Locations, features, and objects. In L. Itti, G. Rees, \& J. K. Tsotsos (Eds.), Neurobiology of attention (pp. 35-41). San Diego: Academic Press.

Shomstein, S., \& YAnTis, S. (2002). Object-based attention: Sensory modulation or priority setting? Perception \& Psychophysics, 64, 41-51.

Shomstein, S., \& Yantis, S. (2004). Configural and contextual prioritization in object-based attention. Psychonomic Bulletin \& Review, 11, 247-253.

Tipper, S. P., Weaver, B., Jerreat, L. M., \& Burak, A. L. (1994). Object-based and environment-based inhibition of return of visual attention. Journal of Experimental Psychology: Human Perception \& Performance, 20, 478-499.

Vecera, S. P. (1994). Grouped locations and object-based attention: Comment on Egly, Drive, and Rafal (1994). Journal of Experimental Psychology: General, 123, 316-320.

VECERA, S. P. (2000). Toward a biased competition account of objectbased segregation and attention. Brain \& Mind, 1, 353-384.

Vecera, S. P., \& Behrmann, M. (2001). Attention and unit formation: A biased competition account of object-based attention. In T. F. Shipley \& P. J. Kellman (Eds.), From fragments to objects: Segregation and grouping in vision (pp. 145-180). Amsterdam: North-Holland.

Vecera, S. P., \& Flevaris, A. V. (2005). Attention control parameters following parietal-lobe damage: Evidence from normal subjects. $\mathrm{Neu}$ ropsychologia, 43, 1189-1203.

Weisstein, N., \& HaRris, C. S. (1974). Visual detection of line segments: An object-superiority effect. Science, 186, 752-755.

Williams, A., \& Weisstein, N. (1978). Line segments are perceived better in a coherent context than alone: An object-line effect in visual perception. Memory \& Cognition, 6, 85-90.

Yantis, S., Schwarzbach, J., Serences, J. T., Carlson, R. L., Steinmetz, M. A., Pekar, J. J., \& Courtney, S. M. (2002). Transient neural activity in human parietal cortex during spatial attention shifts. Nature Neuroscience, 5, 995-1002.

Yantis, S., \& Serences, J. T. (2003). Cortical mechanisms of spacebased and object-based attentional control. Current Opinion in Neurobiology, 13, 187-193.

\section{NOTE}

1. Although we utilize the terminology used by Posner (1980) to describe the processes involved in shifts of attention, we remain neutral on whether there are specific neural mechanisms for each (e.g., a "neural disengager").

(Manuscript received January 24, 2005; revision accepted for publication August 28, 2006. ) 